\title{
Pathophysiological and Psychosocial Study in Patients With Functional Vomiting
}

\author{
Yiming Zhao, $M D^{1}$, Meiyun Ke, MD ${ }^{1 *}$, Zhifeng Wang, $M D^{1}$, Jing Wei, $M D^{2}$, Liming Zhu, MD ${ }^{1}$, Xiaohong Sun, MD ${ }^{1}$ and \\ Jing Zhang, MD ${ }^{1}$ \\ Departments of ${ }^{1}$ Gastroenterology, ${ }^{2}$ Psychology, Peking Union Medical College Hospital, Chinese Academy of Medical Sciences, Beijing, China
}

\author{
Background/Aims \\ To explore clinical patterns, predisposing factors, psychosocial aspects and the possible pathogenesis in Functional Vomiting \\ (FV) patients.
}

\section{Methods}

Ten healthy subjects and 19 FV patients participated in this study. Gastrointestinal symptoms and psychological state were evaluated by questionnaires, including Zung self-rating anxiety and depression scale and Eysenck personality questionnaire. Cutaneous electrogastrography, perfusion nutrition load test and intragastric pressure were performed in patients. Perfusion nutrition load test and intragastric pressure were also performed in healthy subjects.

\section{Results}

FV involved young female predominantly (4 male, 15 female; age $25.8 \pm 8.4$ years). Postprandial vomiting soon after meal without self-induced maneuver was the most common pattern of FV. Prevalence for overlaps between FV and functional dyspepsia was high (84.2\%). Emotional changes and stress contributed to the development of FV. Prevalence of abnormal psychological status and personality in patients with FV was high (83.3\% and $47.1 \%)$. Patients with FV had significant postprandial gastric dysrhythmia, impaired gastric accommodation and enhanced gastric sensitivity. There were significant correlations between clinical presentations, gastric function and psychological states.

\section{Conclusions}

Patients with FV had abnormal psychological status, gastric dysmotility and hypersensitivity, which indicated that both of peripheral and central abnormalities could contribute to the pathogenesis of FV.

(J Neurogastroenterol Motil 2010;16:274-280)

\section{Key Words}

Vomiting, Pathophysiology, Psychosocial factors

Received: April 2, 2010 Revised: July 10, 2010 Accepted: July 11, 2010

(c) This is an Open Access article distributed under the terms of the Creative Commons Attribution Non-Commercial License (http://creativecommons. org/licenses/by-nc/3.0) which permits unrestricted non-commercial use, distribution, and reproduction in any medium, provided the original work is properly cited.

*Correspondence: Meiyun Ke, MD Department of Gastroenterology, Peking Union Medical College Hospital, Chinese Academy of Medical Sciences, Beijing 100-730, China

Tel: +86-10-6525-5006, Fax: +86-10-6525-5052, E-mail: kemypumch2006@yahoo.com.cn

Financial support: None.

Conflicts of interest: None. 


\section{Introduction}

Functional gastrointestinal disorders (FGIDs) are highly prevalent throughout the world. ${ }^{1}$ The absence of anatomic or biochemical markers for FGIDs and the limited number of clinical trials that have been performed, make them a challenge to diagnose. Functional gastroduodenal disorders, one of FGIDs, are classified into 4 categories: functional dyspepsia, belching disorders, functional nausea and vomiting disorders (comprising chronic idiopathic nausea, functional vomiting $[\mathrm{FV}]$ and cyclic vomiting syndrome) and the rumination syndrome. ${ }^{2}$

According to the Rome III criteria, FV is defined as recurrent, unexplained vomiting at least once per week that is not cyclical and lacks an organic basis. ${ }^{3}$ Although $\mathrm{FV}$ is a fairly rare disorder and has been under-investigated, it has been increasingly recognized that this condition can be highly disabling. ${ }^{4}$ Additional studies that investigate the epidemiology and clinical presentation, as well as treatment trials and most importantly mechanistic studies are desperately needed. Psychogenic vomiting was used to describe chronic nausea and vomiting for which there was no apparent cause. ${ }^{5}$ Currently there is no evidence to support an association between any psychiatric disorder and chronic, unexplained nausea and vomiting. ${ }^{4}$ However, stress and psychosocial factors can act as modulators via the brain-gut axis to influence clinical presentation and outcome, which suggests that the association between $\mathrm{FV}$ and psychosocial aspects needs to be further recognized and investigated. ${ }^{6}$

We present a study of consecutive patients admitted to gastrointestinal (GI) clinic in Peking Union Medical College Hospital with chronic vomiting of unknown etiology. The study aims to explore clinical patterns, predisposing factors, psychosocial aspects and gastric sensorimotor function in $\mathrm{FV}$ patients, and to improve recognition of pathogenesis, diagnosis and management of this disorder.

\section{Materials and Methods}

\section{Study subjects}

A total of 10 healthy subjects (HS) (2 male, 8 female; age $30.2 \pm 7.7$ years) and $19 \mathrm{FV}$ patients ( 4 male, 15 female; $25.8 \pm$ 8.4 years) participated in this 8-month (2008.10-2009.6) study consecutively. None of the HS reported symptoms or a history of GI disease or drug allergies, nor were they taking any medication.
All patients fulfilled the Rome III criteria ${ }^{2}$ that must include all of the following: (1) on average, 1 or more episodes of vomiting per week; (2) absence of criteria for an eating disorder, rumination or major psychiatric disease according to Diagnostic and Statistical Manual of Mental Disorders, fourth edition (DSM-IV) and (3) absence of self-induced vomiting and chronic cannabinoid use, and absence of abnormalities in the central nervous system or metabolic diseases to explain the recurrent vomiting. Criteria fulfilled for the past 3 months, with symptom onset at least 6 months before diagnosis. All patients underwent a structured, psychiatric interview as well as a psychological and behavioral history to rule out overt eating disorders, such as anorexia nervosa or bulimia. All patients had undergone previously basic diagnostic testing including complete blood count, electrolyte, fasting glucose, liver and renal function, upper GI barium study and esophagogastroduodenoscopy to exclude structural disorders. The study protocol was approved by the Institutional Ethics committee. Informed consent was obtained from all patients and HS.

\section{Methods}

\section{1) Questionnaires}

Demographic and clinical data were recorded by questionnaires which included vomiting symptoms, overlapping symptoms, psychological states (Zung self-rating anxiety and depression scale $[\mathrm{SAS} / \mathrm{SDS}]),{ }^{7,8}$ social stress (stressful life events or abuse history), and Eysenck personality questionnaire (EPQ). ${ }^{9}$

\section{2) Cutaneous electrogastrography}

Measurements were made using a DIGITRAPPER EGG ${ }^{\mathrm{TM}}$ and the accompanying computerized data analysis package (Synectics Medical Inc, Stockholm, Sweden). Electrogastrography (EGG) was recorded for 30 minutes in fasting state in the morning after an overnight fast and thereafter for another $60 \mathrm{mi}-$ nutes after the test meal. Subjects were requested to intake the test meal within 10 minutes. The test meal consisted of $80 \mathrm{~g}$ of instant noodles, $50 \mathrm{~g}$ of ham and $400 \mathrm{~mL}$ of water. The total energy was 470 kcal. Obtained EGG parameters included dominant frequency (DF), dominant power (DP), \% normal rhythm (\%N), $\%$ bradygastria $(\% \mathrm{~B}), \%$ tachygastria $(\% \mathrm{~T})$ and power ratio (PR). DF was defined as the frequency appearing with peak power value of spectra, whereas DP was the power value observed at DF. Defined range of normal rhythm was 2.4-3.7 cpm. PR was the ratio of the postprandial DP divided by the fasting DP. PR $<1$ was considered as abnormal and likely a poor motor response to the meal. The data of HS were from our previous 
study. ${ }^{10}$

\section{3) Perfusion nutrition load test and intragastric pre- ssure}

Subjects were received perfusion nutrition load test (P-NLT, through nasal-gastric tube) with a constant rate of $50 \mathrm{~mL} / \mathrm{min}$ $(0.75 \mathrm{kcal} / \mathrm{mL})$ in the morning after an overnight fast. ${ }^{11}$ Percentages of protein, carbohydrate and lipid in nutrition liquid were $13 \%, 48 \%$ and 39\%, respectively. Meanwhile, intragastric pressure (IGP) was recorded to assess the sensitivity to gastric distention. ${ }^{11}$ Visual analogue scale $(0-10)$ was used to evaluate satiety during NLT.

\section{Statistical methods}

All data were analyzed by SPSS17.0 software packet. The differences were considered statistically significant when a p-value $<0.05$ was obtained. All measurement data were reported as mean $\pm \mathrm{SD}$. $T$ tests were used to compare differences between 2 groups. Categorical data were analyzed using $\chi^{2}$ test. The correlation of symptoms and gastric function was analyzed by $\chi^{2}$ or Fisher's exact test. The Pearson's correlation analysis was used to evaluate associations among psychological factors and gastric function.

\section{Results}

\section{Clinical characteristics of FV}

Age In FV patients (4 male, 15 female), the age at onset of the first episode ranged from 15 to 44 years (mean, 24 years; median 21 years).

Duration The duration of the FV at the time of consultation ranged from 6 to 109 months (mean, 25 months; median, 13

Table 1. The Prevalence of Overlaps in 19 Functional Vomiting Patients

\begin{tabular}{lc}
\hline \multicolumn{1}{c}{ Overlaps } & $\mathrm{n}(\%)$ \\
\hline GERD & $10(52.6)$ \\
FD & $16(84.2)$ \\
CC & $10(52.6)$ \\
GERD +FD & $8(42.1)$ \\
GERD+CC & $6(31.6)$ \\
FD+CC & $8(42.1)$ \\
GERD +FD +CC & $5(26.3)$ \\
None & $0(0.0)$
\end{tabular}

GERD, gastroesophageal reflux disease; FD, functional dyspepsia; CC, chronic constipation. months).

Severity The severity of FV at the time of the consultation was deemed mild if patients did not have major impairment in daily life function; moderate, if patients had intermittent disruptions in activity; and severe if patients had chronically impaired daily functioning. ${ }^{1}$ Of 19 patients, 1 had mild, 8 had moderate and 10 had severe FV. One patient required nasogastric intubation and enteral nutrition. Six (31.6\%) were disabled and could not maintain their jobs or study.

Patterns of FV All 19 patients had 1 or more episodes of vomiting per week and $78.9 \%$ of them vomited every day. Postprandial vomiting occurred in $94.7 \%$ of these patients. The length of emesis often ranged from minutes to hours. Vomiting occurred without self-induced maneuver. Some patients reported oro-pharyngeal discomfort soon after meals, which could be lessened by vomiting. The vomitus consisted of food initially, sometimes changing to gastric juice and bile. Only 1 patient reported blood streaks and none of them reported vomiting with retained food after overnight fasting.

Overlaps Those between FV and other GI diseases occurred in all patients. The prevalences of overlaps with functional dyspepsia (FD), gastroesophageal reflux disease and chronic constipation were $84.2 \%, 52.6 \%$ and $52.6 \%$ respectively. More than $50 \%$ of patients reported 2 or more overlaps (Table 1 ).

Complications Weight loss occurred in $94.7 \%$ of patients and almost half of women reported menstrual disturbance. Other complications included esophagitis $(21.0 \%)$, electrolytes disturbance $(15.8 \%)$ and dental disease $(5.3 \%)$.

Predisposing factors Sixteen patients (84.2\%) identified

Table 2. Related Factors of Vomiting Spells in 19 Functional Vomiting Patients

\begin{tabular}{lcc}
\hline \multirow{2}{*}{ Related factors } & Predisposing & Exacerbating \\
\cline { 2 - 3 } & $\mathrm{n}(\%)$ & $\mathrm{n}(\%)$ \\
\hline Inappropriate diet & $9(47.4)$ & $5(26.3)$ \\
Satiety & $5(26.3)$ & $1(5.3)$ \\
Alcohol & $2(10.5)$ & $3(15.8)$ \\
Spicy/cold/fatty foods & $3(15.8)$ & $1(5.3)$ \\
Emotion change & $11(57.9)$ & $9(47.4)$ \\
Fatigue & $3(15.8)$ & $2(10.5)$ \\
Mental pressure & $6(31.6)$ & $4(21.0)$ \\
Stress & $6(31.6)$ & $1(5.3)$ \\
Menstrual cycle & $1(5.3)$ & \\
Others & $2(10.5)$ & \\
\hline
\end{tabular}

The relieving factors reported were: multiple small meals in 5 (26.3\%), relaxation and distraction in $5(26.3 \%)$ and rest in $1(5.3 \%)$. 
conditions that seemed to trigger the vomiting episodes. The most frequent triggers reported were: emotional change in 11, inappropriate diet in 9, mental pressure in 6, noxious stress in 6 and fatigue in 3 . Only 1 patient contributed vomiting to menstrual cycle.

Almost half the number of patients reported that emotional change was also a contributory factor for their deterioration of vomiting; in turn, relaxation and distraction could help some patients improve their symptoms (Table 2).

\section{Psychosocial aspects of FV}

Environmental stress was common in $\mathrm{FV}$ patients. Negative life events were elicited in 11 (57.9\%) of our patients and 2 $(10.5 \%)$ of them reported physical or emotional abuse history.

Prevalence of abnormal psychological states was high in FV patients $(83.3 \%)$. Ten $(55.6 \%)$ of our patients displayed significant anxiety and $13(72.2 \%)$ with depression.

As for personality factors, 17 patients completed EPQ and 8 $(47.1 \%)$ of them had high scores on neuroticism scale.

\section{Gastric sensorimotor function}

\section{1) EGG}

Sixteen FV patients received both pre- and post-prandial EGG. One FV patient completed fasting study only because of severe postprandial vomiting. Compared to previous data of $\mathrm{HS}$ in our center, ${ }^{10} \mathrm{FV}$ patients had significantly decreased $\% \mathrm{~N}$ and postprandial DF, increased DP and 50\% with abnormal PR, which indicated postprandial dysrhythmia in FV patients (Table $3)$.

Table 3. The Electrogastrography of Functional Vomiting Patients

\begin{tabular}{|c|c|c|c|c|}
\hline & \multicolumn{2}{|c|}{ Preprandial } & \multicolumn{2}{|c|}{ Postprandial } \\
\hline & $\begin{array}{l}\text { Healthy subjects } \\
\qquad(\mathrm{n}=50)\end{array}$ & $\begin{array}{l}\text { Functional vomiting } \\
\qquad(\mathrm{n}=17)\end{array}$ & $\begin{array}{l}\text { Healthy subjects } \\
\quad(\mathrm{n}=50)\end{array}$ & $\begin{array}{l}\text { Functional vomiting } \\
\qquad(\mathrm{n}=16)\end{array}$ \\
\hline$\% \mathrm{~N}$ & $85.20 \pm 1.70$ & $68.40 \pm 26.40^{\mathrm{a}}$ & $86.00 \pm 2.10$ & $65.00 \pm 21.70^{\mathrm{b}}$ \\
\hline$\% \mathrm{~B}$ & $7.70 \pm 1.50$ & $8.20 \pm 13.90$ & $8.20 \pm 1.30$ & $12.20 \pm 12.40$ \\
\hline$\% \mathrm{~T}$ & $5.70 \pm 1.20$ & $11.40 \pm 13.80$ & $7.90 \pm 1.80$ & $16.30 \pm 13.40^{\mathrm{a}}$ \\
\hline $\mathrm{DF}(\mathrm{cpm})$ & $2.90 \pm 0.02$ & $3.30 \pm 2.00$ & $3.10 \pm 0.02$ & $2.40 \pm 1.20^{\mathrm{a}}$ \\
\hline $\mathrm{DP}(\mathrm{dB})$ & $59.80 \pm 20.40$ & $195.80 \pm 210.40^{\mathrm{a}}$ & $107.00 \pm 37.20$ & $252.20 \pm 239.20^{\mathrm{a}}$ \\
\hline
\end{tabular}

$\% \mathrm{~N}, \%$ normal rhythm; \%B, \% bradygastria; \% T, \% tachygastria; DF, dominant frequency; cpm, counts per minute; DP, dominant power; dB, decibel. The data of HS were from previous study in our center. ${ }^{10}$ ${ }_{\mathrm{p}}^{\mathrm{p}}<0.05,{ }^{\mathrm{b}} \mathrm{p}<0.01$ compared to healthy subjects.

A

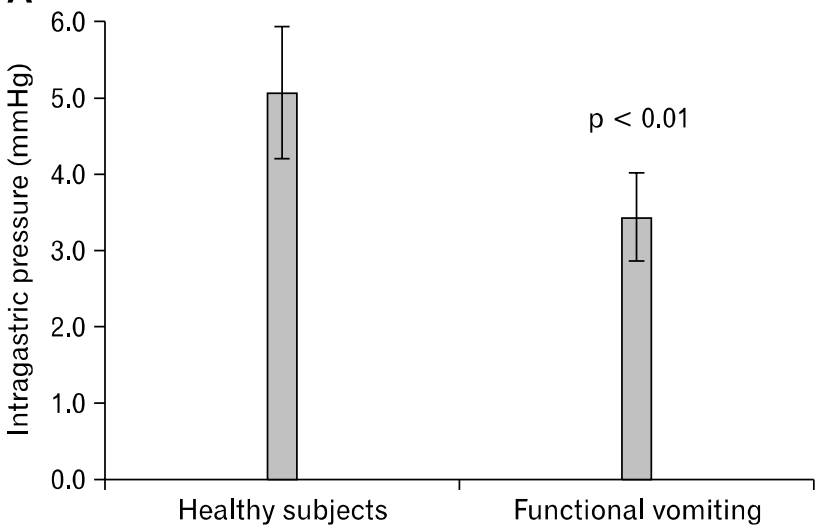

B

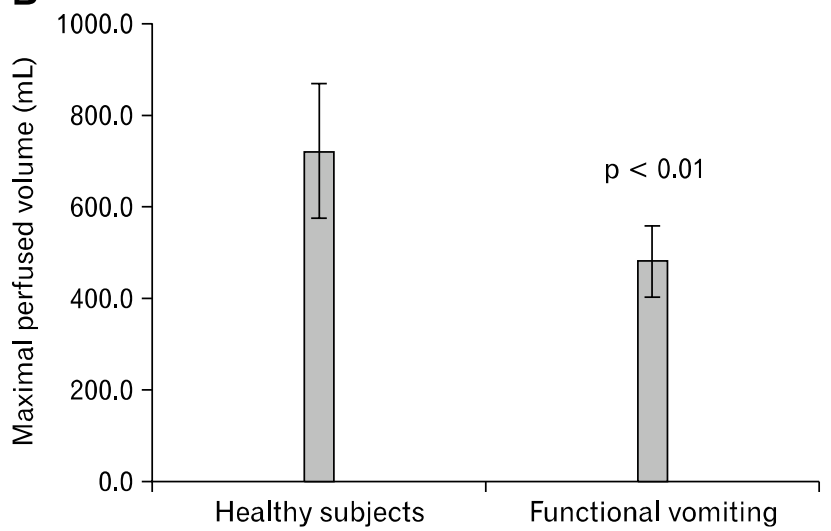

Figure 1. The intragastric pressure (IGP) and the perfused volume of healthy subjects (HS) and functional vomiting (FV) patients at the maximal satiety. (A) Compared to HS, FV patients had significantly decreased IGP at the maximal satiety $(5.1 \pm 1.2 \mathrm{vs} 3.4 \pm 1.0 \mathrm{mmHg})$, indicating enhanced hypersensitivity. (B) Compared to HS, maximal perfused volume in FV patients was significantly decreased (722.0 $\pm 206.2 \mathrm{vs} 481.3 \pm 140.0$ $\mathrm{mL}$ ), which indicated that impaired gastric accommodation could involve in the pathogenesis of FV. 


\section{2) P-NLT and IGP}

Ten HS and $13 \mathrm{FV}$ patients received both P-NLT and IGP test. Two FV patients completed P-NLT only. Compared to $\mathrm{HS}$, the maximal perfused volume and the IGP of FV patients was significantly decreased, indicating impaired accommodation and hypersensitivity in $\mathrm{FV}$ patients (Fig. 1). ${ }^{11,12}$

\section{Relationships among the symptoms, gastric function and psychological factors}

We found significant correlation between the severity of vomiting and $\% \mathrm{~N}$, as well as the prevalence of nausea and PR. Anxiety (SAS) and neuroticism (EPQ-N) could predict the amount of overlaps. The postprandial DF, DP and the MPV were related to the scores of SAS, SDS and EPQ-N (Table 4-6).

\section{Discussion}

Vomiting was a common complaint and a study from USA showed a total of $2 \%$ reported vomiting monthly or more

Table 4. Relationships Between Psychiatric States, Personality and Overlaps in Functional Vomiting Patients

\begin{tabular}{lcc}
\hline & \multicolumn{2}{c}{ The amount of overlaps } \\
\cline { 2 - 3 } & $\mathrm{r}$ & $\mathrm{p}$-value \\
\hline SAS & 0.550 & $0.022^{\mathrm{a}}$ \\
SDS & 0.394 & 0.118 \\
EPQ-N & 0.496 & $0.043^{\mathrm{a}}$
\end{tabular}

$r$, contingency coefficient; SAS and SDS, self-rating anxiety and depression scale; EPQ-N, neuroticism scale in Eysenck personality questionnaire. ${ }^{\mathrm{a}} \mathrm{p}<0.05$. frequently. ${ }^{13}$ However, FV was not common after an extensive evaluation. ${ }^{14}$ Muraoka et al ${ }^{15}$ reported that 59 patients with vomiting of unclear etiology were divided into five arbitrary patterns of vomiting (continuous, postprandial, irregular, nausea and self-induced). The pattern of vomiting in $\mathrm{FV}$ was just like that in the postprandial group described by Muraoka et al and was unmistakably different from other vomiting disorders as rumination syndrome, bulimia, gastroparesis and cyclic vomiting syndrome. In our study, the majority of FV patients could stop vomiting themselves, indicating that behavior therapy could be beneficial for the $\mathrm{FV}$ patients.

Olden and $\mathrm{Crowell}^{4}$ found that $\mathrm{FV}$ patients were much more likely to have a FGID than patients with vomiting of organic diseases. Recent studies have revealed that overlaps are common in patients with FGIDs and about two thirds have symptoms of multiple disorders at the same time. ${ }^{16,17}$ There were significant correlation between overlaps and psychological factors. ${ }^{18,19}$ Our study also found that anxiety and neuroticism could predict the prevalence of overlaps.

It is now widely accepted that dysfunction of the bidirectional neural pathways between the brain and the gut ('brain-gut axis') at any level can contribute to the various symptoms experienced by FGID patients. ${ }^{20}$ From the brain level, psychological disturbances, for example acute or chronic stress reaction, psychiatric disorders, especially emotional disorders, anxiety disorders and somatoform disorders, may be associated with alterations in the processing of visceral sensation and gut-related autonomic nervous system function, affecting gut motility and sensation. As shown in our study, 11/16 patients cited emotional changes as a triggering factor of the occurrence of their symptom, and almost half the number of patients reported that emotional change was

Table 5. Relationships Between Symptoms and Gastric Sensorimotor Function in Functional Vomiting Patients

\begin{tabular}{|c|c|c|c|c|c|c|c|}
\hline & $\begin{array}{c}\text { Severity of } \\
\text { vomiting }\end{array}$ & Nausea & $\begin{array}{c}\text { Postprandial } \\
\text { distention }\end{array}$ & $\begin{array}{c}\text { Early } \\
\text { satiation }\end{array}$ & $\begin{array}{c}\text { Epigastric } \\
\text { pain }\end{array}$ & $\begin{array}{c}\text { Epigastric } \\
\text { burning }\end{array}$ & Belching \\
\hline & \multicolumn{7}{|c|}{ p-value } \\
\hline \multicolumn{8}{|l|}{ EGG } \\
\hline Pre- $\% \mathrm{~N}$ & 0.452 & 1.000 & 0.662 & 0.153 & 0.593 & 1.000 & 0.050 \\
\hline Post- $\% \mathrm{~N}$ & $0.020^{\mathrm{a}}$ & 0.615 & 1.000 & 0.126 & 0.596 & 0.633 & 0.315 \\
\hline PR & 0.264 & $0.041^{\mathrm{a}}$ & 0.608 & 1.000 & 0.282 & 0.608 & 1.000 \\
\hline \multicolumn{8}{|l|}{ P-NLT } \\
\hline MPV & 0.626 & 1.000 & 1.000 & 1.000 & 1.000 & 0.400 & 1.000 \\
\hline IGP & 0.874 & 0.462 & 1.000 & 0.192 & 1.000 & 1.000 & 1.000 \\
\hline
\end{tabular}

EGG, electrogastrography; Pre- and Post-\%N, pre- and post-prandial \% normal rhythm; PR, power ratio; P-NLT, perfusion nutrition load test; MPV, maximal perfused volume; IGP, intragastric pressure. ${ }_{\mathrm{a}}^{\mathrm{p}}<0.05, \mathrm{p}$-value is for $\mathrm{r}$ (contingency coefficient). 
Table 6. Relationships Between Psychiatric States, Personality and Gastric Sensorimotor Function in Functional Vomiting Patients

\begin{tabular}{|c|c|c|c|c|c|c|}
\hline & \multicolumn{2}{|c|}{ SAS } & \multicolumn{2}{|c|}{ SDS } & \multicolumn{2}{|c|}{ EPQ-N } \\
\hline & $\mathrm{r}$ & $\mathrm{p}$-value & $\mathrm{r}$ & $\mathrm{p}$-value & $\mathrm{r}$ & p-value \\
\hline \multicolumn{7}{|c|}{ Preprandial EGG } \\
\hline$\% \mathrm{~N}$ & -0.271 & 0.328 & -0.069 & 0.806 & -0.395 & 0.146 \\
\hline DF & 0.300 & 0.278 & 0.181 & 0.518 & 0.369 & 0.175 \\
\hline $\mathrm{DP}$ & 0.311 & 0.260 & 0.035 & 0.901 & 0.336 & 0.221 \\
\hline \multicolumn{7}{|c|}{ Postprandial EGG } \\
\hline$\% \mathrm{~N}$ & -0.315 & 0.252 & -0.354 & 0.196 & -0.449 & 0.093 \\
\hline DF & -0.626 & $0.012^{\mathrm{a}}$ & -0.648 & $0.009^{\mathrm{a}}$ & -0.483 & 0.068 \\
\hline DP & 0.556 & $0.031^{\mathrm{a}}$ & 0.508 & 0.053 & 0.539 & $0.038^{\mathrm{a}}$ \\
\hline PR & 0.335 & 0.222 & 0.413 & 0.126 & 0.321 & 0.243 \\
\hline \multicolumn{7}{|l|}{ P-NLT } \\
\hline MPV & -0.642 & $0.018^{\mathrm{a}}$ & -0.713 & $0.006^{\mathrm{a}}$ & -0.252 & 0.407 \\
\hline IGP & 0.034 & 0.920 & -0.046 & 0.893 & 0.278 & 0.407 \\
\hline
\end{tabular}

SAS and SDS, self-rating anxiety and depression scale; EPQ-N, neuroticism scale in Eysenck personality questionnaire; r, contingency coefficient; EGG, electrogastrography; \%N, \% normal rhythm; DF, dominant frequency; DP, dominant power; PR, power ratio; MPV, maximal perfused volume; IGP, intragastric pressure.

${ }^{a} \mathrm{p}<0.05$

also a contributory factor for their deterioration of vomiting, while relaxation and distraction could help some patients improve their symptoms. Negative life events and abuse history were also found in patients in our study. This was observed in many other studies. ${ }^{6}$ Some researchers suggested that personality traits may explain the association between abuse and FGIDs. Abuse may induce the expression of abnormal personality traits that in turn leads to FGIDs. ${ }^{21}$ There was also an increased prevalence of abnormal psychological states in clinic patients with FGIDs (in our study, $83.3 \%$ ) compared with HS (20\%). ${ }^{6}$ One study reported higher levels of depression in $\mathrm{FV}$ patients. ${ }^{15}$ Another study showed that neuroticism was increased in patients with FGIDs. ${ }^{22}$ Our study found that about half FV patients showed high neuroticism, which meant that they were more likely than the average to experience feelings such as anxiety and depression and cope poorly with environmental stress. ${ }^{9}$ Neuroticism may explain the association between abuse and $\mathrm{IBS}^{22}$ and predict treatment outcome in FGID patients. ${ }^{23}$ Our findings pointed to the possibility that psychosocial factors could play an important role in pathogenesis of FV. Accordingly, psychosocial factors and psychiatric disorders should be considered in the evaluation and treatment of FV patients. It may also be a direction to improve further study, that to include professional psychiatrists/psychologists to thoroughly evaluate life events and abuse and do possible interventions.

Patients with unexplained nausea and vomiting had abnormal EGG and the improvement of EGG was related to the relief of the symptoms. ${ }^{14,24}$ The characteristic abnormality observed in EGG pattern in FV patients was postprandial gastric dysrhythmia, such as decreased $\% \mathrm{~N}$ and $\mathrm{DF}$, an absence of the normal postprandial power increase after the test meal. The observed correlation of decreased $\% \mathrm{~N}$ with severity of vomiting and abnormal PR with nausea revealed that the decreased postprandial electrical response activity was related to the symptoms in $\mathrm{FV}$ patients. Our study found that decreased DF was associated with the scores of SAS, SDS and EPQ-N, which suggested that psychological factors could involve in gastric dysrhythmia. Our findings also showed correlations between impaired gastric accommodation and the level of SAS/SDS, which was also supported by previous studies. ${ }^{25,26}$ However, our study did not reveal the relationship between gastric hypersensitivity and psychological status. The relationship between psychological status and gastric dysfunction now is still controversial. Although we assumed that P-NLT was more objective than drink nutrition load test and more physiological than barostat, ${ }^{11}$ it has not been recognized as golden standard for assessment of gastric accommodation and sensation. Further studies are necessary to confirm the validation of this method. It is unclear if psychosocial factors mainly determine healthcare seeking or have a more direct influence on GI sensorimotor function in FGIDs. Recent epidemiological and neurobiological studies have provided increasing evidence for the second hypothesis. ${ }^{6}$ Central nervous system structures, which integrate sensory information or regulate autonomic output to the viscera, largely overlap with regions involved in emotional regu- 
lation in the brain. ${ }^{20}$ This might provide an explanation for the positive relationship between abnormal psychological status and gastric function in $\mathrm{FV}$ patients.

In summary, this is, to our knowledge, the first study to describe the clinical pattern, pathophysiology, psychosocial factors and their relationships in patients with FV. Gastric dysmotility and hypersensitivity may have a key role in the causation of $\mathrm{FV}$. Gender, age, stress and psychosocial factors can act as modulators via the brain-gut axis or other approaches to influence clinical presentation and outcome of FV. Both of peripheral and central abnormalities could contribute to the pathogenesis of $\mathrm{FV}$. Further studies are warranted to gain more insight into the complex interaction of peripheral abnormalities and brain centers.

\section{Acknowledgements}

The authors would like to thank staffs from the Department of Gastroenterology in the Peking Union Medical College Hospital for their assistance.

\section{References}

1. Drossman DA. The functional gastrointestinal disorders and the Rome III process. Gastroenterology 2006;130:1377-1390.

2. Tack J, Talley NJ, Camilleri M, et al. Functional gastroduodenal disorders. Gastroenterology 2006;130:1466-1479.

3. Talley NJ. Functional nausea and vomiting. Aust Fam Physician 2007;36:694-697.

4. Olden KW, Crowell MD. Chronic nausea and vomiting: new insights and approach to treatment. Curr Treat Gastroenterol 2005; 8:305-310

5. Hederos CA. Psychogenic vomiting. Lancet 1992;339:1228.

6. Levy RL, Olden KW, Naliboff BD, et al. Psychosocial aspects of the functional gastrointestinal disorders. Gastroenterology 2006;130: 1447-1458.

7. Zung WW. A rating instrument for anxiety disorders. Psychosomatics 1971;12:371-379.

8. Zung WW. A self-rating depression scale. Arch Gen Psychiatry 1965;12:63-70

9. Eysenck H, Eysenck S. The manual of the Eysenck Personality Questionnaire. London: Hodder \& Stoughton 1975.

10. Chen YM, Ke MY, Wang ZF, Zhang XL, Pan GZ. Electrogastrogrephy in fifty healthy subjects. Chin J Dig 2000;20:227-229.

11. Zhang J, Sun XH, Ke MY, Wang ZF. Perfusion nutrition load test in assessment of gastric accommodation and sensitivity in functional dyspepsia patients - a preliminary study [abstract]. Korean J Neuro- gastroenterology Motil 2009;15:29.

12. Tack J, Caenepeel P, Piessevaux H, Cuomo R, Janssens J. Assessment of meal induced gastric accommodation by a satiety drinking test in health and in severe functional dyspepsia. Gut 2003;52:1271-1277.

13. Talley NJ, Zinsmeister AR, Schleck CD, Melton LJ 3rd. Dyspepsia and dyspepsia subgroups: a population-based study. Gastroenterology 1992;102:1259-1268.

14. Abell TL, Familoni B, Voeller G, et al. Electrophysiologic, morphologic, and serologic features of chronic unexplained nausea and vomiting: lessons learned from 121 consecutive patients. Surgery 2009; 145:476-485.

15. Muraoka M, Mine K, Matsumoto K, Nakai Y, Nakagawa T. Psychogenic vomiting: the relation between patterns of vomiting and psychiatric diagnoses. Gut 1990;31:526-528.

16. Corazziari E. Definition and epidemiology of functional gastrointestinal disorders. Best Pract Res Clin Gastroenterol 2004;18: 613-631.

17. Drossman DA, Li Z, Andruzzi E, et al. U.S. householder survey of functional gastrointestinal disorders. Prevalence, sociodemography, and health impact. Dig Dis Sci 1993;38:1569-1580.

18. Lee SY, Lee KJ, Kim SJ, Cho SW. Prevalence and risk factors for overlaps between gastroesophageal reflux disease, dyspepsia, and irritable bowel syndrome: a population-based study. Digestion 2009; 79:196-201.

19. Song ZQ, Ke MY, Wang ZF, Liu XH, Fang XC. Dyspeptic symptomatology and pathogenesis of functional dyspepsia with and without overlapping symptoms: a comparative study. Chin J Gastroenterol 2006;11:458-461.

20. Van Oudenhove L, Demyttenaere K, Tack J, Aziz Q. Central nervous system involvement in functional gastrointestinal disorders. Best Pract Res Clin Gastroenterol 2004;18:663-680.

21. Talley NJ, Boyce PM, Jones M. Is the association between irritable bowel syndrome and abuse explained by neuroticism? A population based study. Gut 1998;42:47-53.

22. Tanum L, Malt UF. Personality and physical symptoms in nonpsychiatric patients with functional gastrointestinal disorder. J Psychosom Res 2001;50:139-146.

23. Tanum L, Malt UF. Personality traits predict treatment outcome with an antidepressant in patients with functional gastrointestinal disorder. Scand J Gastroenterol 2000;35:935-941.

24. Geldof H, van der Schee EJ, van Blankenstein M, Grashuis JL. Electrogastrographic study of gastric myoelectrical activity in patients with unexplained nausea and vomiting. Gut 1986;27:799-808.

25. Geeraerts B, Vandenberghe J, Van Oudenhove L, et al. Influence of experimentally induced anxiety on gastric sensorimotor function in humans. Gastroenterology 2005;129:1437-1444.

26. Van Oudenhove L, Vandenberghe J, Geeraerts B, et al. Relationship between anxiety and gastric sensorimotor function in functional dyspepsia. Psychosom Med 2007;69:455-463. 